Monatsschr Kinderheilkd

2020 • 168 (Suppl 3):S154-S162

https://doi.org/10.1007/s00112-020-00946-4

Online publiziert: 5 . August 2020

(c) Springer Medizin Verlag GmbH, ein Teil von Springer Nature 2020

\section{Die Heilpädagogische Abteilung der Wiener Universitätskinderkli- nik nahm bald nach ihrer Gründung 1911 eine zentrale Position im Wie- ner Fürsorgesystem ein, die sie für Jahrzehnte behielt. Behörden und Privatpersonen wandten sich mit dem Wunsch nach Beobachtung ihrer schutzbefohlenen Minderjährigen, nach Diagnose und Maßnahmen- empfehlung an die Abteilung, die durch ihren Gründer, den österrei- chischen Kinderarzt Erwin Lazar (1877-1932), und später durch Hans Asperger (1906-1980) geprägt war. Das Ergebnis der heilpädagogischen Untersuchung hatte großen Einfluss auf das Leben der PatientInnen, ins- besondere wenn es um Fragen der Fremdunterbringung ging.}

Da zuletzt Aspergers Involvierung in das NS-Regime im Fokus der wissenschaftlichen Auseinandersetzung stand (vgl. die Beiträge von Czech und - als Reaktion auf seine Forschungen - Maleczek et al., Waldhauser et al. und Tatzer im vorliegenden Heft), rückte ein anderer zentraler Punkt vorübergehend in den Hintergrund. Dieser betrifft die Bedeutung der Heilpädagogischen Abteilung für die Beurteilung, Kategorisierung und Bewertung Minderjähriger und die damit zusammenhängenden Folgen für die Betroffenen selbst sowie für die Verfestigung und Verbreitung heilpädagogischer

Dieser Beitrag stammt aus der Dissertation der Verfasserin: "[...] ist ohne lückenlose Führung gefährlich und auch selbst gefährdet." Die Heilpädagogische Abteilung der Wiener Universitäts-Kinderklinik von 1911 bis 1949. Konzepte und Kontinuitäten.

Ina Friedmann

Wissenschaftsbüro Innsbruck e. V., Innsbruck, Österreich

\title{
Die Heilpädagogische Abteilung der Wiener Universitätskinder- klinik zwischen 1911 und 1977
}

Theorien, die an der Abteilung entwickelt worden waren, in anderen gesellschaftlichen und institutionellen Bereichen.

\section{Gründung, Aufgabe und Akteurlnnen}

Die Heilpädagogische Abteilung der Universitätskinderklinik in Wien wurde im Dezember 1911 unter Zusammenwirken des neuen Klinikvorstands Clemens Pirquet (1874-1929) und dem ersten Abteilungsleiter Erwin Lazar eröffnet. Sie war zunächst als Begutachtungseinrichtung für Kinder und Jugendliche gedacht, die vor der Aufnahme in den Pestalozzi-Verein zur Förderung des Kinderschutzes und der Jugendfürsorge standen [16, S. 161]. Lazar war seit 1906 ärztlich für den Verein tätig, der gleich anderen Wohltätigkeitsvereinen die Aufgaben der damals noch nicht verstaatlichten Kinder- und Jugendfürsorge wahrnahm sowie neben Horten und Tagesstätten auch Heime betrieb. ${ }^{1}$ Seine pädiatrische Ausbildung wurde durch die psychiatrische Arbeitserfahrung bei dem bekannten Psychiater und Nobelpreisträger Julius Wagner-Jauregg (1857-1940) ergänzt. Damit und mit seinen Tätigkeiten als Sachverständiger für Jugendliche am Wiener Landesgericht ab 1910, für das Wiener Jugendamt nach dessen Einrichtung 1916, in der Reorganisation der niederösterreichischen Landes-Besserungsanstalten ab 1912 sowie als Konsulent im Ministerium für soziale Verwaltung ab 1919 war die

\footnotetext{
1 Zum Pestalozziverein sowie generell den Wiener Kinderschutzvereinen um 1900: Malleier [20].
}

Ausrichtung seiner Heilpädagogik eng verbunden. $^{2}$ Nach Lazars Auffassung war Heilpädagogik ein „direkter Abkömmling der allgemeinen Psychiatrie“. Sie sollte aber darüber hinausgehend unter Zusammenwirken von Pädiatrie, Psychiatrie, Psychologie und Pädagogik die endogenen und exogenen Faktoren, die für die beanstandeten Verhaltensweisen ausschlaggebend waren, individuell gewichten und, darauf aufbauend, geeignete Maßnahmen für die PatientInnen empfehlen. Neben Fürsorgevereinen und jenen Institutionen, für die Lazar beratend tätig war (insbesondere Jugendamt, Erziehungsanstalten und Jugendgericht), wandten sich bald weitere Personen und Einrichtungen mit dem Wunsch nach Begutachtung Minderjähriger an die Abteilung. Hierzu zählten Eltern, praktische und Fachärzte/Fachärztinnen, Normal-, Hilfs- und Sonderschulen, die Polizei sowie Einrichtungen der Fremdunterbringung (Internate, Kinder- und Erziehungsheime; [16, S. 161-163]).

Diese von Lazar etablierten Tätigkeitsbereiche und Kooperationen blieben über Jahrzehnte hinweg bestehen, und trotz der sukzessiven Errichtung vergleichbarer Beobachtungsräume in Einrichtungen der genannten Organe behielt die Heilpädagogische Abteilung ihre zentrale Position in diesem Gefüge. Hier wurden besonders jene Minderjährigen aufgenommen, deren Diagnostizierung als zu diffizil beispielsweise in der Ambulanz, die der Abteilung angegliedert war, oder eben in anderen heilpädagogischen Begutachtungsräumen angesehen

\footnotetext{
${ }^{2}$ Archiv der Universität Wien (UAW), Personalakt (PA) Erwin Lazar.
} 
wurde. ${ }^{3}$ Auch als auffällig wahrgenommenes Verhalten, das prinzipiell längere Beobachtungszeit erforderte, führte zur stationären Aufnahme. Dies konnte das Benehmen betreffen, aber ebenso die Abklärung körperlicher Zustandsbilder wie etwa Anfälle unterschiedlicher Art.

\section{》) Heilpädagogik stellte 1911 ein innovatives interdisziplinäres Forschungsfeld dar}

Personell war die Abteilung durch lange Kontinuitäten geprägt. Lazar stand der Abteilung für 20 Jahre vor; nach seinem überraschenden Tod infolge einer Operation übernahm die Ärztin und Abteilungsmitarbeiterin Valerie Bruck (1894-1961) von 1932 bis 1935 die Leitungstätigkeit. Sie arbeitete bereits seit 1923 an der heilpädagogischen Abteilung und war seit 1928 nebenamtlich als Sachverständige am Wiener Jugendgericht sowie als ärztliche Leiterin der Erziehungsanstalt Hirtenberg tätig. Abgesehen davon war sie in der St.-Lukas-Gilde aktiv, deren Mitglied auch ihr Nachfolger Hans Asperger war. ${ }^{4}$ Während Lazar zahlreiche Fachpublikationen veröffentlichte, in denen er seine Sichtweisen und Konzepte darlegte und immer wieder die praktische Arbeit an der Abteilung beschrieb, ${ }^{5}$ trat Bruck publikatorisch kaum in Erscheinung. Durch die austrofaschistische Gesetzgebung war mit der Doppelverdienerverordnung bereits 1933 begonnen worden, verheiratete Frauen aus dem öffentlichen Dienst zu drängen. ${ }^{6}$

3 Als solche nannte Lazar 1923: Schutzheim für die männliche Jugend (Wien 13, St. Veitgasse), Schutzheim für die weibliche Jugend (Wien 18, Staudtgasse), Frauenspital in Klosterneuburg, Niederösterreich, polizeiliches Jugendheim (Wien 3, Juchgasse) sowie "das Jugendgericht in seinen Untersuchungs- und Strafhaftabteilungen“ $[16$, S. 162].

${ }^{4}$ Fragebogen, Magistratsdirektion der Stadt Wien, 22.09.1945, Wiener Stadt- und Landesarchiv (WStLA), PA Valerie Bruck. Zur St.-LukasGilde sowie katholischen Eugenik in Österreich: Löscher [19].

${ }^{5}$ Ein Publikationsverzeichnis findet sich bei Fadinger [9].

6 Verordnung der Bundesregierung vom 15.12.1933 über den Abbau verheirateter weiblicher Personen im Bundesdienste und an-
Entsprechend dem allgemeinen politischen Klima sollten Frauen allerdings generell weitgehend von Erwerbsarbeit ausgeschlossen werden. Auch Brucks zuletzt auf ein Jahr erfolgte Bestellung zur unbesoldeten Assistentin mit Stipendium wurde 1935 nicht mehr verlängert. ${ }^{7}$ Von Franz Hamburger (1874-1954), der die Leitung der Kinderklinik nach Pirquets Suizid im Jahr 1930 übernommen hatte, wurde daraufhin der seit 1931 an der Kinderklinik und seit 1932 an der Heilpädagogischen Abteilung arbeitende Hans Asperger eingesetzt. Er leitete die Abteilung bis 1957.

Da sich drei weitere Beiträge dieses Sonderhefts dezidiert mit Asperger und seinen biografischen Stationen auseinandersetzen, ist an dieser Stelle der Fokus auf die Kontinuitäten, die sein Wirken auszeichnen, zu legen. Als Leiter der Heilpädagogischen Abteilung war er nicht nur in drei politischen Systemen in derselben Funktion tätig. Er war außerdem in der Nachkriegszeit akademischer Lehrer jener HeilpädagogInnen, die den in den 1950er-Jahren gegründeten heilpädagogischen Landeseinrichtungen vorstanden: Irene Plaß (Steiermark), Ingeborg Judtmann (Salzburg), Erwin Schmuttermeier (Niederösterreich) und Franz Wurst (Kärnten). ${ }^{8}$ Neben der auf

dere dienstrechtliche Maßnahmen, BGBI. 1933, Stück 174,Nr. 545.

7 Die letzte Bestellung Brucks zur unbesoldeten Assistentin mit Forschungsstipendium als Leiterin der Abteilung erfolgte vom 01.05.1934 bis zum 30.04.1935. Med. Dek. Akt 895-1933/34, UAW. Ihr Scheiden aus der Abteilung ist mit der austrofaschistischen „Doppelverdienerverordnung" in Verbindung zu bringen, denn obwohl sie als unbesoldete Assistentin nicht direkt unter die Verordnung fiel, ,unterstützte das austrofaschistische Regime ein frauenfeindliches Klima, in dem Erwerbsarbeit von Frauen, insbesondere von verheirateten Frauen, auf jeder Ebene und nicht nur im Bundesdienst angefeindet wurde" [6,S.276].

8 Einen Überblick über die zeitnahen Gründungen sowie die Aufgaben und Ausrichtungen dieser Institutionen gibt der Abschnitt zu den österreichischen "Heilpädagogischen Landschaften" in dem Sammelband von DietrichDaum et al. [8]. - Lediglich Maria NowakVogl, Tirol, war keine Schülerin Aspergers, doch berief auch sie sich in der Praxis kontinuierlich auf Aspergers 1952 erstmals erschienene Monografie Heilpädagogik. diesem Weg erfolgten Verbreitung von Aspergers wissenschaftlichen Theorien in Österreich ist besonders die in diesen Fällen ebenfalls langjährige Prägung der jeweiligen Einrichtungen durch ihre Leitungspersonen hervorzuheben. Diese hierarchischen Konzeptionen, die zudem über Jahrzehnte Bestand hatten, sind als unentbehrliche Eckpfeiler der regionalen Fürsorgesysteme zu sehen.

Nachdem Asperger 1957 zum Vorstand der Innsbrucker Kinderklinik berufen worden war, übernahm Paul Kuszen (1920-2016) die Leitung der Heilpädagogischen Abteilung. Er war 1955, nach 2-jähriger Praxis als Kinderarzt in Niederösterreich, als Assistent an die Abteilung gekommen, an der er bereits von 1951 bis 1953 nebenberuflich als Gastarzt gearbeitet hatte. Neben der Abteilungsleitung übernahm Kuszen von Asperger auch die Lehrtätigkeit, ab dem Wintersemester 1957/1958 hielt er allerdings vorübergehend ausschließlich „Schwesternkurse“ und „Fürsorgerinnenkurse“ am pädagogischen Institut ab. Darüber hinaus war er „Fachschularzt an den Hilfsschulen der Stadt Wien".9 Auch als Asperger 1962 als Vorstand an die Wiener Kinderklinik zurückkehrte, behielt Kuszen die Abteilungsleitung - bis 1978. Wie weitreichend die heilpädagogische Arbeit der Abteilung auch in der zweiten Hälfte des 20. Jh. war, wird an folgender Aussage Aspergers über Kuszen 1966 greifbar:

Er [Kuszen] ist für die Klinik absolut unentbehrlich, wobei noch betont werden muß, daß seine Arbeit weit über den Rahmen der Klinik in das Gefüge der sozialen Arbeit Wiens und Österreichs hinausgreift. In dieser seiner Arbeit verzehrt er sich vollkommen, so daß keine Zeit für die Produktion wissenschaftlicher Publikationen bleibt, allerdings ist er dafür auch nicht sehr talentiert, während alle seine übrige Arbeit auf höchstem Niveau steht. ${ }^{10}$

Entsprechend Aspergers Einschätzung publizierte Kuszen, wie Valerie Bruck, tatsächlich kaum - vor allem keine eigenständigen wissenschaftlichen Theorien.

\footnotetext{
9 Lebenslauf, o.D., UAW, PA Paul Kuszen.

10 Asperger an Bundesministerium für Unterricht, 17.03.1966, UAW, PA Paul Kuszen.
} 
Über die genaue Zahl des Abteilungspersonals sind keine Aufstellungen vorhanden, bereits seit den 1930er-Jahren gehörten neben den ÄrztInnen allerdings - fast durchgängig - auch Psychologinnen zu den MitarbeiterInnen. Bei der Abteilungseröffnung waren regulär ausgebildete weltliche Kinderkrankenschwestern eingesetzt worden. Doch bald wurde die Notwendigkeit erkannt, diese für den Umgang mit den heilpädagogischen PatientInnen zu schulen. Die ab den 1920er-Jahren an der Abteilung tätige Schwester Viktorine Zak (1897-1944), die zudem zwei Publikationen über ihre Arbeit verfasste, wurde von Asperger besonders geschätzt [5, 9, $23,24]$.

\section{Bruch und Kontinuität}

Um die Bedeutung eines Aufenthalts an der Heilpädagogischen Abteilung für die PatientInnen nachvollziehen zu können, ist zunächst die bereits begonnene Auseinandersetzung mit jenen AkteurInnen, die das Funktionieren der Einrichtung gewährleisteten und ihr den inhaltlichen Rahmen gaben, zu vertiefen. Durch den Blick auf die Personen, die die Heilpädagogische Abteilung prägten, kann bei Einbeziehung ihrer wissenschaftlichen Publikationen jeweils eine Ausrichtung bzw. Schwerpunktsetzung ihrer Herangehensweise an die Heilpädagogik beobachtet werden. Damit ist nicht die Fokussierung eines bestimmten Arbeitsgebietes gemeint, wie es bei Lazar die Erforschung der Ursachen der „Dissozialität“ Minderjähriger oder bei Asperger jene der ,autistischen Psychopathie“, später als Asperger-Syndrom bezeichnet, war. Vielmehr ist die Orientierung an sozialen oder biologistischen Denkkonzepten gemeint, die für die Sichtweise auf die (angeblich) zugrunde liegenden Ursachen kindlichen und jugendlichen Verhaltens ausschlaggebend waren. Während Lazar für die individuelle Bewertung der Einflüsse endogener und exogener Faktoren eintrat (z. B. [17, S. 2-3, 18]), war für Asperger primär die Veranlagung der Minderjährigen ausschlaggebend (z. B. [3, S. 262]). Dass er dennoch starkes Gewicht auf das „Milieu“ legte und entsprechend häufig

Monatsschr Kinderheilkd 2020 · 168 (Suppl 3):S154-S162

https://doi.org/10.1007/s00112-020-00946-4

(c) Springer Medizin Verlag GmbH, ein Teil von Springer Nature 2020

\section{Friedmann}

\section{Die Heilpädagogische Abteilung der Wiener Universitätskinderklinik zwischen 1911 und 1977}

\section{Zusammenfassung}

Die Heilpädagogische Abteilung der Kinderklinik fungierte bald nach ihrer Eröffnung 1911 als zentrale Verteilungseinrichtung für „verhaltensauffällige" Kinder und Jugendliche im Wiener Fürsorgesystem. Dieser Beitrag fokussiert auf die Funktionsweise dieser Einrichtung, wobei über die politischen Zäsuren des 20. Jh. hinweg gezeigt wird, dass lange Kontinuitäten sowohl in personeller als auch inhaltlicher Hinsicht prägend waren. Unterstützt durch Krankengeschichten der Abteilung werden Beurteilungsschemata sichtbar gemacht und die Position der Abteilung im Gefüge der Agenturen der Fürsorge diskutiert. Dieser mehrdimensionale Einblick in das komplexe Thema der Institutionenund PatientInnengeschichte trägt dazu bei, die Bedeutung von Fürsorgekooperationen aus verschiedenen Perspektiven zu sehen. Dadurch gelingt es, in der Auseinandersetzung um biografische Aspekte historischer Persönlichkeiten auch den Raum, in dem diese wirkten, sowie die davon primär Betroffenen, hier also die PatientInnen, in die Betrachtung einzubeziehen.

Schlüsselwörter

Kindliches Verhalten - Kinder- und Jugendfürsorge - Gesundheitseinrichtungen . Fremdunterbringung · Geschichte

\section{The ward for Therapeutic Pedagogy at the Vienna University Children's Clinic between 1911 and 1977}

\section{Abstract}

Soon after its opening in 1911, the ward for Therapeutic Pedagogy of the pediatric hospital became a central institution for distributing children and adolescents seen as maladjusted within the Vienna welfare system. This article focuses on the functioning of this institution, showing how staff and their scientific theories were continuing beyond the political turning points of the twentieth century. Supported by patient records of the department, assessment schemes are made visible as well as the position of the department within the system of welfare agencies. This multidimensional insight into the complex field of the institutional and patient histories helps to see the importance of welfare cooperation from various perspectives. It also enables a portrayal of those primarily concerned, in this case the patients, in the discussion of biographical aspects of historical personalities.

Keywords

Child behavior - Children and youth welfare . Healthcare facilities - Out of home care . History dessen Veränderung empfahl, erscheint nur auf den ersten Blick als Paradoxon. Dies ist durch seine Ansicht erklärlich, dass es bei den verschiedenen Problematiken gerade aufgrund der Bedeutung der Veranlagung besonders guter, auch im Sinn starker, erzieherischer Einflüsse bedurfte. Sein Leitsatz war „strikte Führung“ zu „optimaler Leistung “ [4, S. 355, 2, S. 13]. Der Normalisierungsanspruch der heilpädagogischen Wissenschaft, der eng mit Leistungsprinzipen verknüpft war, wird hieran deutlich.

Unterschiedliche Herangehensweisen dürfen allerdings nicht den Eindruck erwecken, dass daraus auch wesentliche
Unterschiede in der Maßnahmensetzung resultierten. Minderjährige, die entweder als „schwererziehbar“ galten oder aus sozialen Gründen nicht in ihrer Ursprungsfamilie leben konnten (etwa aufgrund von Misshandlung) oder sollten (etwa, wenn Erziehungsberechtigte als „erziehungsuntüchtig“ angesehen wurden), wurden in Institutionen eingewiesen, die durch gewaltförmige Erziehungsmethoden geprägt waren. Das bedeutet, dass, abgesehen von der autoritären Regulierung sämtlicher Lebensbereiche, also der „Gewalt über den Alltag“, auch die Anwendung psychischer und physischer Gewalt verbreitet war. Die Stigmatisie- 
rung von Kindern und Jugendlichen als „schwererziehbar“ erfolgte dabei institutionell und unwiderruflich - und hatte wesentlichen Einfluss auf das weitere Leben der Betroffenen. Lautete das heilpädagogische Urteil „schwererziehbar“, „psychopathisch“ oder ähnlich, war ein oft mehrjähriger Aufenthalt in Erziehungsheimen die Folge.

\section{》) Aspergers Leitsatz war die "strikte Führung" zu „optimaler Leistung"}

Neben der in diesem Kontext zu stellenden Frage nach den Beurteilungsmustern, die der Charakterisierung Minderjähriger als „schwererziehbar“ zugrunde liegen, ist die Verantwortung des Staates und der in seinem Auftrag handelnden Personen und Institutionen anzusprechen: Erfolgt von staatlicher Seite ein Eingriff in das Privatleben seiner BürgerInnen, in diesem Fall primär im Sinn von „Kindsabnahmen“ durch das Jugendamt und seine Agenturen, sollte damit grundlegend die Sicherung des körperlichen und seelischen Wohls der betroffenen Kinder und Jugendlichen verbunden sein. Dass der Staat diese Aufgabe nicht wahrnahm, zeigen die mittlerweile über ganz Österreich vorliegenden Berichte über Gewalt in Kinder- und Erziehungsheimen.

Wie dieser institutionelle Kreislauf vielfach ausgesehen hat, soll ein Beispiel verdeutlichen. Es wurde 1952 gemeinsam mit weiteren biografischen Skizzen von Insassen der Bundesanstalt für Erziehungsbedürftige Kaiserebersdorf in der Zeitung Der Abend infolge der dortigen „Zöglingsrevolte“ publiziert. Die Anstalt Kaiserebersdorf, die als „Endstation“ unter den Erziehungseinrichtungen galt, stellte ein Jugendgefängnis dar, dessen Zustände 1952 zum Aufbegehren der Internierten geführt hatten. Die in dem Bericht wiedergegebene Aussage des Direktors von Kaiserebersdorf spricht für sich: „Von den Buben, die hier sind, hat schon jeder was am Kerbholz. Alle sind sie vom Jugendrichter zu uns eingewie- sen worden." ${ }^{11}$ So auch ein 9-jähriger Bub, dessen Familienbiografie mit jener unzähliger in Fürsorgeerziehung stehender Kinder im Nachkriegsösterreich identisch ist - außerehelich geboren, die Mutter alleinstehend und für den Familienunterhalt als Arbeiterin verantwortlich:

Da sich die Mutter um ihn nicht kümmern konnte, kam er in Fürsorgeerziehung, wurde von einem "Heim" ins andere geschoben. Siebenmal brach er aus und wurde auf dem Weg zur Mutter aufgegriffen. Als pädagogische Maßnahme steckte man ihn in ein Heim, das recht weit vom Wohnort der Mutter entfernt ist. Das hätte doch das Heimweh des kleinen Poldi heilen sollen, nicht wahr? Aber Poldi zeigte für diese erzieherische Weisheit kein Verständnis. Wieder machte er sich auf die Reise zur Mutter. Auf dem langen Weg brach er wo ein, wahrscheinlich um sich Proviant zu verschaffen.

Das heilpädagogische Gutachten spricht von „völlig ungehemmt ablaufenden Affekten" und von einer "tierhaften" Gewandtheit. „Die Aufgreifung ging immer unter ungeheuren Exzessen vor sich. "Poldi wehrte sich nämlich verbissen und verzweifelt, wenn man ihn wieder von der Mutter weg in ein Heim bringen wollte. ${ }^{12}$

Das Gutachten über den 9-jährigen Buben entstand vermutlich nicht an der Heilpädagogischen Abteilung, die Sprache ist aber die der Institution: Abgesehen von der häufigen Bezugnahme auf „Affektabläufe“ ist der Ausdruck „tier[l]haft“ nicht nur anderen oft in der Diagnostik der Abteilung verwendeten typisierenden Begriffen ähnlich, sondern wurde dort als solcher zwischen 1937 und 1944 11-mal PatientInnen zugeschrieben ${ }^{13}$, darunter auch einmal als „tierlhafte Gewandtheit“ (1938) - davor wurde er

\footnotetext{
${ }^{11}$ Ernst Epler, Jugend hinter Gittern. Kaiserebersdorf von innen gesehen. Der Abend, 24.11.1952.

12 Ernst Epler, Jugend hinter Gittern. Kaiserebersdorf von innen gesehen. Der Abend, 24.11.1952.

${ }^{13}$ Es ist aufgrund der erstmaligen Dokumentation nach seiner Leitungsübernahme davon auszugehen, dass Asperger diesen Ausdruck in das Begriffsinventar der Abteilung eingeführt
} hatte. nicht benutzt. Zwar stellt dieses Beispiel keinen (bekannten) Fall der Heilpädagogischen Abteilung dar, dennoch wurde es aus mehreren Gründen ausgewählt: Es gab ein heilpädagogisches Gutachten, das Jugendgericht war involviert, und es verdeutlicht den Blick auf Minderjährige aus prekären Familienverhältnissen auch in den Nachkriegsjahren.

Von der Wiener Heilpädagogischen Abteilung wurden bis 1949 keine Patienten nach Kaiserebersdorf überstellt, in 6 Fällen (1931, 1933, 2-mal 1934, 1936, 1937) aber wurde die Unterbringung in dieser Erziehungsanstalt empfohlen. In anderen Fällen zeigen nachträglich den Krankengeschichten beigelegte Korrespondenzen, dass ehemalige Patienten nach Aufenthalten in anderen Heimen in Kaiserebersdorf untergebracht worden waren. Dass diese Kinder zuvor nicht (erneut) an der Heilpädagogischen Abteilung aufgenommen wurden, erklärt sich aus der Möglichkeit ambulanter Untersuchungen, aber insbesondere aus den meist im Rahmen der Anhalteeinrichtungen des Jugendgerichts durchgeführten heilpädagogischen Begutachtungen.

\section{》) In der Beurteilung kam der sozialen Situation der Familien große Bedeutung zu}

Dass dieses Beispiel so aussagekräftig ist, liegt nicht ausschließlich im beschriebenen Umgang mit dem Buben. Darüber hinaus verdeutlicht der Vergleich mit Angaben in einer Krankengeschichte, welche Bedeutung der Familiensituation in der Beurteilung zukam: Als Beispiel kann ein Fall aus dem Jahr 1943 angeführt werden, in dem häufige Fluchtversuche aus einem Internat aus Heimweh zur Aufnahme eines 10-jährigen Buben an der Heilpädagogischen Abteilung geführt hatten. Der Bub war öfter auch unterwegs aufgegriffen und entweder zurück in das jeweilige Schülerheim oder zu seiner Familie gebracht worden. ${ }^{14}$ Im Befund der Heilpädagogischen Abteilung wurde festgehalten:

\footnotetext{
14 WStLA, 1.3.2.209.1.A47, 1943 A-M, ohne Prot.- oder Ambulanzkarten-Nr.
} 
Es handelt sich um einen sehr nervösen, affektiv besonders leicht erregbaren, unausgeglichenen Knaben. Daher reagierte er auch auf den Versuch, ihn von zuhause wegzugeben und in einem Pensionat unterzubringen, mit einem übermässig starken, unbeherrschbaren Heimweh, das ihn wiederholte Fluchtversuche unternehmen liess.... Wir sind daher der Meinung, dass man die weitere Entwicklung dieses sensiblen Kindes gefährden würde, wenn man das Verbleiben in einem Pensionat gegenwärtig zu erzwingen versuchte. ${ }^{15}$

Der wichtigste Unterschied in diesen beiden Fällen ist der Familienhintergrund der Buben: ein uneheliches Kind einer Marktarbeiterin gegenüber einem Arztsohn aus traditionellen Familienverhältnissen. Staatlich verordnete Heimunterbringung gegenüber Internatsaufenthalt. In beiden Fällen Fluchten aus Heimweh. Kaiserebersdorf gegenüber der Rückkehr in die Familie.

\section{》) Als „Locktypen“ bezeichnete weibliche sexuelle Miss- brauchsopfer verdeutlichen die Diagnosepraxis}

Bezugnehmend auf die zu besprechenden Kontinuitäten und Brüche ist ein bedeutender Unterschied zwischen Lazar und Asperger in der Veränderung ihrer Sichtweisen zu thematisieren: Lazar erprobte etwa seine Ideen zur Gruppierung von Minderjährigen nach unterschiedlichen Gesichtspunkten (Diagnose, Körperbau) in Anstalten und veränderte diese bei Scheitern nicht nur in der Praxis, sondern publizierte dies auch. Asperger dagegen publizierte nach 17jähriger Leitungstätigkeit und der Veröffentlichung zahlreicher wissenschaftlicher Arbeiten seine Monografie Heilpädagogik erstmals 1952. Darin finden sich Ansätze, die er bereits in den 1930erJahren in gleicher Form an der Abteilung praktizierte - etwa, was den Umgang mit sexueller Gewalt gegen weib-

15 Befund an Leitung der Oberschule in St., 10.11.1943, WStLA, 1.3.2.209.1.A47, 1943 A-M, ohne Prot.-oder Ambulanzkarten-Nr. liche Minderjährige angeht - und die er offensichtlich als bewährt und gültig eingestuft hatte. Der von ihm eingeführten Charakterisierung mancher weiblicher Opfer sexueller Gewalt als „passive Locktypen", auch in seiner Heilpädagogik dargelegt [3, S. 262], begegnet man in den Akten erstmals 1936, also ein Jahr nach Übernahme der Abteilungsleitung. ${ }^{16} \mathrm{Be}-$ reits davor waren Mädchen als „Locktypen" bezeichnet worden, was der typisierenden Diagnosepraxis der Abteilung entsprach. ${ }^{17}$ Die Verschärfung durch die Zuschreibung angeblicher Passivität hatte naturgemäß auch in einem breiteren Kontext Bedeutung: Mit dieser Schuldverschiebung ging einerseits eine Rückwirkung dieser Sichtweise auf die betreffenden Minderjährigen selbst einher (sowohl was psychische Auswirkungen als auch weitere Maßnahmensetzungen betraf), andererseits konnte sie auch im juristischen Diskurs von Bedeutung sein. ${ }^{18}$

\section{Die Heilpädagogische Abteilung als Institution: Funktion und Struktur}

Wie bei jeder Auseinandersetzung mit einer Institution ist auch im Fall der Heilpädagogischen Abteilung der theoretische Blick auf ihre Funktionsweise zur Verortung in einem breiteren Kontext unerlässlich. Strukturelle Faktoren sind

\footnotetext{
16 WStLA, 1.3.2.209.1.A47, 1936 A-M, ohne Prot.- oder Archiv-Nr. In den Neuauflagen des Buchs Heilpädagogik. Einführung in die Psychopathologie des Kindes für Ärzte, Lehrer, Psychologen und Fürsorgerinnen von Asperger [3] in den Jahren 1956 (2. neu bearbeitete und erweiterte Auflage, S. 269), 1961 (3. neu bearbeitete und erweiterte Auflage, S. 285), 1965 (4. unveränderte Auflage, S. 285) und 1968 (5. unveränderte Auflage, S. 285) findet sich der Passus zu den „passiven Locktypen” unverändert.

17 Erstmals 1922, in diesem Jahr gleich mehrfach: WStLA, 1.3.2.209.1.A47, 1922 N-Z, Prot.Nr. 64; 77 und 95; WStLA, 1.3.2.209.1.A47, 1922 A-M, Prot.-Nr. 107.

18 Beispiele für die konkrete Beurteilung von betroffenen Kindern und Jugendlichen unterbleiben aus Platzgründen. Für eine Auseinandersetzung mit der Thematik: Friedmann [13]. Hier auch zur Einstufung der Glaubwürdigkeit minderjähriger Missbrauchsopfer, die zentral für die Identifizierung tatsächlicher "gewissenloser Verführer"war [3, S.233].
}

ebenso zu berücksichtigen wie soziale, gesellschaftliche und politische. Eine Institution existiert nicht losgelöst von diesen Elementen, sondern steht im Kontakt, teilweise in Abhängigkeit, jedenfalls in Wechselbeziehung, zu ihnen. Darüber hinaus sind ausgehend vom "Ressourcenmodell“" des langjährig in Wien tätigen US-amerikanischen Historikers Mitchell Ash Wissenschaft und Politik auch hier als Ressourcen füreinander zu verstehen, die im Kontakt mit der jeweils anderen Sphäre ihre Interessen vertreten und verfolgen [1]. Es gilt daher, die Heilpädagogische Abteilung als Institution zu charakterisieren und nach ihren strukturellen Eigenschaften ebenso zu fragen wie nach ihrer Funktionsweise und ihren Beziehungen zu ihren PatientInnen, aber auch zu anderen Einrichtungen.

Dem kanadischen Soziologen Erving Goffman (1922-1982) folgend ist die Abteilung als "totale Institution“ zu verstehen, deren wesentliches Merkmal die Vereinigung aller Lebensbereiche unter einer Autorität ist: Arbeitszeit (hier Lernzeit), Freizeit und Rückzugszeit fanden am selben Ort statt und waren genau reglementiert. Die in diesen Zeitsegmenten auszuführenden Tätigkeiten wurden von einer Gruppe, deren Mitglieder sich in derselben Position befanden, also den PatientInnen, gemeinsam verrichtet, was einem übergeordneten Ziel dienen sollte. Das war in diesem Fall die abschließende Beurteilung der PatientInnen, die aus der Beobachtung in dem dazu geschaffenen Raum zu resultieren hatte. Hinzu kam die „Vermischung“ der Lebensbereiche, die einen Vergleich des Verhaltens in einem Bereich mit dem in einem anderen ermöglichte. Ihr Lebensraum für einen bestimmten Zeitraum, den der Aufenthaltsdauer, „stellt[e] für sie eine Art Welt für sich dar" [15, S. 15, 17, 44]. Diese Aussage hat umso mehr Gültigkeit, wenn die Isolierung der PatientInnen von ihrem persönlichen, privaten Umfeld berücksichtigt wird. Aber auch die Gegenüberstellung der Lebensrealitäten der beiden Gruppen PatientInnen und Personal verdeutlicht das Gesagte [15, S. 18-19]: Die Heilpädagogische Abteilung war für die Aufenthaltsdauer der Raum, in dem sich das Leben der dort beobachteten Minderjährigen abspielte, und zwar rund um die 
Uhr und mit strikt reguliertem, seltenem Kontakt zur Außenwelt. Auf der anderen Seite stand das Personal, das sich in diesem Lebensraum als Arbeitsraum bewegte. Für diese Gruppe gab es Zeiten außerhalb der Einrichtung; sie konnte sie verlassen, zu bestimmten Zeiten, aber auch allgemein, wenn man ihrer überdrüssig war, und sie war nicht zuletzt für die Etablierung, Überwachung und Durchsetzung der geltenden Regeln zuständig. In ihrem Handlungsspielraum lagen Kulanz und Härte im Umgang mit Verstößen. Auch die Durchführung der „Aufnahmeprozedur" ist in diesen Kontext zu setzen, die Rituale wie die Erhebung der Anamnese, Fotografieren, körperliche Untersuchung und Verzeichnung der Ergebnisse beinhaltete. Damit im Zusammenhang steht der Verlust der „Identitätsausrüstung “, der die Verweigerung persönlicher Gegenstände unterschiedlicher Art, die zur Aufrechterhaltung des eigenen Selbst benötigt werden, bezeichnet. Ein weiterer wichtiger Kontrollmechanismus lag im Vorenthalten von Informationen [15, S. 18-20, 27, 30]. Dies konnte bereits im Vorfeld der Aufnahme geschehen, wenn Erziehungsberechtigte oder Behörden die Minderjährigen im Unklaren darüber ließen, dass oder auch weswegen sie in die Heilpädagogische Abteilung eingewiesen wurden. Aber auch an der Abteilung selbst, insbesondere wenn es um die Entlassungsorte ging, wurde PatientInnen nicht transparent kommuniziert, welcher Unterbringungsort (und weswegen) im Anschluss an den Stationsaufenthalt vorgesehen war. Informationen wurden auch im Rahmen des Anamnesegesprächs gewonnen und verzeichnet, „besonders die diskreditierenden - über den sozialen Status und die Vergangenheit der Insassen ... und in einem dem Personal zur Verfügung stehenden Dossier zusammengestellt."Auch die Gesprächssituationen während des Aufenthalts erfüllten einen Zweck: Sie sollten die Perspektive der PatientInnen sichtbar machen, aber zugleich den ÄrztInnen ermöglichen, die Persönlichkeit der Minderjährigen zu beurteilen. Neben dieser klinischen Funktion ist allerdings das erweiterte Setting zu berücksichtigen: „Zu solchen Gelegenheiten muß der Insasse Fakten und Gefühle, die seine Person be- treffen, ihm bisher unbekannten Zuhörern offenbaren “ $[15$, S. 33]. Reaktion und Verhalten dienten dann der Beurteilung. Obwohl dies unerlässlich für das Funktionieren der heilpädagogischen Begutachtung war, handelte es sich um eine spezielle Situation für die PatientInnen. Wurden bestimmte Informationen oder Details im als „Examen“ bezeichneten Gespräch ausgespart, wurde dies von den ÄrztInnen registriert. Ihnen standen die bereits (vielfach im Vorfeld) gesammelten Informationen zur Verfügung, was bedeutet, dass die Entscheidung für oder gegen die Wiedergabe bestimmter Inhalte von den fragenden Personen direkt in die Beurteilung einbezogen werden konnte. Der Verlust der Entscheidung darüber, mit wem man persönliche Informationen in welcher Art teilen will, bedeutet zugleich einen Kontrollverlust über die eigene Privatsphäre [15, S. 37].

\section{》) Patientlnnen wurden bei Nichterfüllung normierender Kriterien der korrigierenden Erziehung zugeführt}

Wichtig ist in diesem Kontext das Bewusstsein dafür, dass es sich bei der Heilpädagogischen Abteilung um eine Station handelte, die nicht mit anderen Stationen der Kinderklinik vergleichbar war bzw. ist. Das bedeutet, dass gesammelte Daten über Familienangehörige nicht allein einer somatischen Bewertung von (familiären) Vorerkrankungen oder der Artikulation von Misshandlungsverdacht dienten, sondern eine klassifizierende Beurteilung ermöglichen sollten. Alleinstehende Mütter beispielsweise wurden im heilpädagogischen Kontext einer anderen Kategorisierung unterzogen als auf den anderen Kinderstationen. Die Besprechung ihres „Lebenswandels“ etwa findet sich nur in Anamnesen der Heilpädagogischen Abteilung. Auch ging es nicht um eine Einschätzung eines möglicherweise auftretenden Misshandlungsverdachts; dieser war bei betroffenen PatientInnen bereits im Vorfeld geäußert worden und hatte vielfach zur Aufnahme an der Heil- pädagogischen Abteilung geführt. Dort sollte er verifiziert oder verneint werden.

Die Beschreibung der Heilpädagogischen Abteilung als totale Einrichtung im Sinn Goffmans dient dazu, den Blick über die engen institutionellen Grenzen, in denen diese Organisationsform sinnvoll erscheinen kann, hinaus zu erweitern: auf die Bedeutung, die dieses Modell für die ihm zugeführten Individuen hatte oder haben konnte. Die Einrichtung selbst konnte zweifelsfrei ohne die derartige Ordnung ihren Zweck nicht erfüllen. Dieser Zweck war nach Kriegsende 1918 auch von staatlicher Seite definiert worden: „Alle sozialpolitischen Maßnahmen sollten ,produktiv' sein.“ Das von Julius Tandler (1869-1936), Anatom und Wiener Stadtrat für das Wohlfahrtswesen, „geforderte ,sichere wissenschaftliche Wissen' für alle konkreten Entscheidungen im Bereich der Fürsorgeerziehung sollten die Gutachten der heilpädagogischen Ärzte erzeugen“ [22, S. 34]. Zentral war nach dem Sozialhistoriker Reinhard Sieder das Konzept der „schuldhaften Verwahrlosung“, das (nicht nur) im Wiener Fürsorgekontext leitend war. Er beschreibt, dass Tandler überzeugt war, „dass sozial untüchtige und arme Eltern für die laufende Pflege und Erziehung von Kindern untauglich seien, ,minderwertige ' Anlagen auf ihre Kinder vererbten und so zur Verbreitung ungünstiger (,negativer') Anlagen innerhalb der ,Rasse` beitrügen. Deshalb schien ihm der durch die Fürsorgerin erbrachte Befund der relativen Untüchtigkeit von Eltern in Elternschaft und Haushalt schon Grund genug, ihnen ein Kind, mehrere oder alle Kinder ,abzunehmen' und in Kinderheimen einer Ersatzerziehung zuzuführen" [22, S. 40-41].

Aber abgesehen von der Frage nach den gesellschaftlichen und staatlichen Bedürfnissen, die eine Institution wie die Heilpädagogische Abteilung zu einer unhinterfragten Notwendigkeit werden ließen, darf mit dieser Kenntnis ein anderer Faktor nicht unberücksichtigt bleiben, nämlich die Auswirkungen auf die diesem Komplex Unterworfenen. Die PatientInnen wurden nach normierenden Kriterien beurteilt und bei Nichtentsprechen einer korrigierenden 
Erziehung zugeführt. Die Heilpädagogische Abteilung ist hier in einem Kontext mit den übrigen Agenturen der Minderjährige betreffenden Überwachungsund Korrekturinstitutionen $\mathrm{zu}$ sehen. Mit Sieder ist sie als eines mehrerer Subsysteme zu verstehen, die das System der Wiener Kinder- und Jugendfürsorge konstituierten [22, S. 42-43]. Die Heilpädagogische Abteilung untersuchte die "schweren Fälle“, vielfach im Auftrag der anderen Einrichtungen, aber auch aus wissenschaftlichem Interesse in Eigeninitiative, und stellte durch ihre wissenschaftlich fundierten Diagnosen und darauf beruhenden Maßnahmenempfehlungen eine zentrale Drehscheibe für als "auffällig" eingestufte Minderjährige dar.

\section{》) Einweisungen in Erziehungs- heime trugen zum langjährigen Fortbestehen von Korrektur- erziehungseinrichtungen bei}

Die besondere Position, die der Abteilung dennoch zukommt, manifestiert sich durch ihre Funktion in der wissenschaftlichen Rechtfertigung von Einrichtungen der „Korrekturerziehung“. Während sie von der Organisation her ein ähnlich hierarchisches Gepräge aufwies wie etwa Kinder- und Erziehungsheime, gibt es ein wesentliches Unterscheidungsmerkmal: Der Aufenthalt an der Heilpädagogischen Abteilung war von Beginn an auf einen kürzeren Zeitraum beschränkt. Auch der Zweck des Aufenthalts unterschied sich maßgeblich, da es nur begrenzt um Korrektur und auch nicht um Bestrafung ging, sondern diese Maßnahmen potenziell aus der heilpädagogischen Untersuchung resultierten sowie von ihr legitimiert und angeordnet wurden. Wichtig ist auch der Blick auf die empfohlenen Einrichtungen zur folgenden Fremdunterbringung in jenen Fällen, in denen diese verfügt oder angeregt wurde. Daraus lässt sich bis zu einem gewissen Grad auf die „inhaltliche Orientierung" der Abteilung schließen, also die Befürwortung oder Ablehnung bestimmter Erziehungskonzepte. Damit einhergehend muss darauf hingewiesen werden, dass die in den Forschungen der letzten Jahre dokumentierten gewaltförmigen Erziehungsmethoden in den (gesamt-)österreichischen Kinderund Erziehungsheimen durch die Inanspruchnahme und Empfehlung seitens der Heilpädagogik so lange fortbestehen konnten.

\section{Prüfung und Beurteilung}

Der Aufenthalt an der Heilpädagogischen Abteilung ist vom Zeitpunkt des Betretens der Institution bis zum Verlassen gewissermaßen als Prüfungssituation anzusehen. Geprüft wurde jeder Aspekt des kindlichen Wesens, vom Aussehen bis zum Gefühlsleben. Die vom französischen Philosophen Michel Foucault (1926-1984) für Disziplinaranstalten beschriebene Bedeutung der „Prüfung“ kann ebenfalls für die Charakterisierung der Begutachtungssituation an der Heilpädagogischen Abteilung gelten: „Die Prüfung kombiniert die Techniken der überwachenden Hierarchie mit denjenigen der normierenden Sanktion. Sie ist ein normierender Blick, eine qualifizierende, klassifizierende und bestrafende Überwachung" [12, S. 238]. Die „überwachende Hierarchie" beeinflusste in diesem Kontext nicht nur das Verhalten der Überprüften, sondern gab die Rahmenbedingungen vor. Vermeintlich „natürliche“ Situationen sollten Gelegenheit geben, das Verhalten der PatientInnen zu beobachten und einzuordnen: Die Disziplinarmacht „setzt sich durch, indem sie sich unsichtbar macht, während sie den von ihr Unterworfenen die Sichtbarkeit aufzwingt" [12, S. 241]. Genau beobachtet wurde daher bereits, wie sich Minderjährige im Moment der Aufnahme verhielten; die „Heimwehreaktion“ wurde als „normal“ oder „abnormal“ eingestuft und stellt vielfach die erste Notiz über das Verhalten eines Kindes in der Krankenakte dar. Begutachtet wurden in der Folge jede Verhaltensweise, jede (unterlassene) Handlung, sämtliche Interaktionen, aber auch die Intelligenz der PatientInnen, ihr Schulwissen, Lern- und Sozialverhalten. Unterschieden wurden "freie“ und Prüfungssituationen. Auch das Verhalten gegenüber den Krankenschwestern fand Eingang in die Krankenakte. Der Begutachtungsprozess sowie das daraus resultierende abschließende Gutachten selbst stellen somit „ein Bündel von Aktivitäten" dar, zu dem neben der Messung der genannten Parameter die Auseinandersetzung mit den bisher entstandenen Akten - ihre Lektüre und Evaluation - zählte [14, S. 18]. Vom Abteilungspersonal gemachte Beobachtungen und Einschätzungen wurden zuverlässig in den PatientInnenakten notiert, welche Informationen allerdings tatsächlich Eingang in den jeweiligen Befund fanden, variierte. Eine Leerstelle in den Krankenakten bildeten Anpassungsstrategien der Betroffenen. Ihr Verhalten wurde zumeist nach vorgefassten Kriterien beurteilt; wenig Augenmerk wurde auf individuelle Bewältigungsstrategien unterschiedlicher Situationen oder Erlebnisse gelegt, wodurch die Normierung, Qualifizierung und Klassifizierung, also die Einpassung individuellen Handelns in ein Codesystem, evident wird.

\section{》) Eine Leerstelle in den Krankenakten bildeten kindliche Anpassungsstrategien}

Das Beispiel eines 13-jährigen Patienten aus dem Jahr 1939 kann verdeutlichen, was damit gemeint ist. Der Jugendliche war vom 07.10.1939 bis zum 11.12.1939 an der Abteilung aufgenommen worden, als Einlieferungsgründe waren auf dem Deckblatt der Krankengeschichte verzeichnet: „Vagieren, Kaffeehausbesuche, Schulschwänzen, [h]omosexuelle Gesellschaft, Selbstmordversuch" ${ }^{19}$ Zwei Jahre zuvor war der Vater des Jugendlichen, zu dem der Patient ein sehr enges Verhältnis gehabt habe, verstorben: „Einige Tage nach dem Tode des Vaters erfuhr er auch erst, dass die Stiefm. nicht seine richtige Mutter sei. Seither wurde sein Verhalten der St.M. gegenüber immer ärger.“20 Das Auffallende an der Krankenakte des Jugendlichen ist, dass der Tod des Vaters und die Falschannahme über die Stief-

\footnotetext{
19 WStLA, 1.3.2.209.1.A47, 1939A-M, ohne Prot.- oder Archiv-Nr.

20 Anamnese, WStLA, 1.3.2.209.1.A47, 1939 A-M, ohne Prot.- oder Archiv-Nr.
} 
mutter als Mutter zwar registriert und in der Anamnese als Ausgangspunkt der Verhaltensschwierigkeiten besprochen, jedoch im Folgenden nicht weiter in die Beurteilung des Patienten einbezogen wurden:

Die Erziehung und Führung eines solchen Menschen ist begreiflicher Weise ungemein schwierig. Es ist ganz falsch, den Buben, der bei seiner Leere mit sich ohnehin nichts rechtes anzufangen weiss, seiner Wege gehen zu lassen, wie das bei seiner Mutter geschehen ist (die Mutter ist viel zu schwach für ihn, hat derzeit auch ganz andere Interessen - Heiratsabsichten usw. - wie wir denn überhaupt über sie recht ungünstige Auskunft erhalten haben). Der Knabe braucht unbedingt eine sehr straffe Führung von aussen, da ihm eben die innere Disziplin so weitgehend fehlt. Er braucht aber auch ein besonderes pädagogisches Verständnis, das seine Konfliktneigung, seine Empfindlichkeiten, die ja nicht bloss Faxen, sondern in seinem Wesen begründet sind, berücksichtigt, den Bogen nicht überspannt und doch fest und konsequent bleibt. Von der Mutter ist, wie schon gesagt, diese Erziehung keineswegs zu erwarten, eine Rückgabe an sie kommt also auf keinen Fall in Frage, wenn der Knabe selbst auch am liebsten zu ihr ginge, weil er dort die meisten Freiheiten hat. $^{21}$

\section{\) Ergebnisse des heilpädago- gischen Gutachtens gingen als objektive Wahrheit in Fürsorgebeschlüsse ein}

Der Patient wurde schließlich in den Haushalt seines Bruders und dessen Frau entlassen, die sich bereit erklärt hatten ihn aufzunehmen und denen erzieherisches Geschick attestiert wurde. Frappierend ist an diesem Fall das Fehlen eines Eingehens auf das Ereignis, das das beanstandete Verhalten auslöste, weshalb er zur Illustration der Bedeutung der Einpassung von Individualität in normierende Schemata herangezogen wurde: Der

${ }^{21}$ Gutachten, 30.11.1939, WStLA, 1.3.2.209.1. A47, 1939 A-M, ohne Prot.- oder Archiv-Nr.
Patient wurde individuell wahrgenommen, und biografische Einschnitte wurden dokumentiert - seine Reaktion darauf aber losgelöst davon normierend bewertet.

Gerade an diesem Fall kann zudem die institutionelle Kompilation der unterschiedlichen Informationen über Minderjährige sichtbar gemacht werden: An die Heilpädagogische Abteilung von der Psychiatrie transferiert, finden sich über den dortigen Aufenthalt zwar keine Schriftstücke, das Jugendamt war aber bereits im Vorfeld in das Leben des Jugendlichen involviert. Von der Abteilung wurde, basierend auf den dort gemachten Beobachtungen, ein Gutachten für das Jugendgericht im die Unterbringung des Patienten betreffenden Pflegschaftsfall erstellt, das die Grundlage für den ebenfalls der Akte beiliegenden Gerichtsbeschluss darstellte. Dieser zeigt in seiner Begründung das Gewicht, das der heilpädagogischen Expertise zukam: Als wissenschaftlicher Ratgeberin wurde ihren Einschätzungen und Empfehlungen nicht nur gefolgt, sondern es kam auch zur Aneignung der Inhalte in der Form, dass eine Kenntlichmachung wiedergegebener Informationen als aus der heilpädagogischen Beurteilung stammend unterblieb und diese als Fakten, als objektive Wahrheit Eingang in den Beschluss fanden. ${ }^{22}$ Dies war generell der Fall bei Dokumenten, die im Fürsorgekontext entstanden und die ihren urteilenden, unauslöschlichen Niederschlag in den Akten erfuhren. Das ist deshalb hervorzuheben, weil dieser Niederschlag die begutachteten Minderjährigen als „papierener Schatten“ zumindest bis zur Volljährigkeit begleitete [7, S. 346]. Konkret bedeutet das, dass nicht nur Abschriften heilpädagogischer Befunde und Gutachten an Behörden und Institutionen weitergeleitet wurden - und gerade bei Minderjährigen in Fürsorgeerziehung zum Anwachsen des Mündelakts und damit auch zur Verdichtung der „Aktenfigur“ eines Kindes beitrugen ${ }^{23}$. Befundabschriften, vielfach

\footnotetext{
22 Beschluss, 15.01.1940, WStLA, 1.3.2.209.1. A47, 1939 A-M, ohne Prot.- oder Archiv-Nr.

${ }^{23}$ Eingehend dazu das Kapitel „Die Mündelakte" in Heimkindheiten. Geschichte der Jugendfür-
}

einschließlich eines Kommentars, wurden außerdem teilweise mehrere Jahre nach dem Abteilungsaufenthalt noch an anfragende Einrichtungen übermittelt.

\section{Schlussbemerkungen}

Zuletzt ist auf die reale Bedeutung der heilpädagogischen Konzepte hinzuweisen, die an der Abteilung entwickelt, erprobt, überprüft, angewendet und schließlich auch weiterverbreitet wurden. Durch die zentrale Position im Wiener Fürsorgesystem erlangten neben der praktischen Tätigkeit auch die theoretischen Ideen der an der Abteilung wirkenden Personen breite Aufmerksamkeit und Gewicht. Foucault folgend kann festgehalten werden: „Im Namen einer dringenden biologischen und historischen Notwendigkeit rechtfertigte sie [die Wissenschaft] die drohend bevorstehenden Staatsrassismen. Sie begründete sie in ,Wahrheit" [11, S. 58]. Deutlich wird an dieser Aussage die Wechselwirkung, die gegenseitige Bedingung von begründendem wissenschaftlichem Wahrheitsanspruch, der - vielfach für den Staat - die Rechtfertigung für Eingriffe in private Lebenswelten entwickelte. Die unterschiedlichen Ausprägungen und Bereiche sind das, was sie sind: unterschiedliche Ausprägungen und Bereiche, bei gleichbleibenden Mechanismen. Das bedeutet, dass die im Nationalsozialismus erfolgten Eingriffe in Körper und Leben von Menschen, die auf als wissenschaftlich untermauert ausgewiesenen Klassifizierungssystemen beruhten, die Extremform dieser wissenschaftlichen Legitimation darstellen, dass aber die Regulierungsmacht des Staats, die Deutungshoheit über Lebensentwürfe nicht erst damit einsetzte. Auch zuvor galt es zunächst, die UntertanInnen eines Souveräns, später die StaatsbürgerInnen $\mathrm{zu}$ beobachten und $\mathrm{zu}$ korrigieren [10, S. 115-116]. Neben den mit der Veränderung der Konzeption von Familie etablierten Moralvorstellungen betraf dies - durchaus mit der Anerkennung bestimmt(er) ausgeformter Familienstrukturen einhergehend bzw. zumin-

sorge und Heimerziehung in Tirol und Vorarlberg von Ralser et al. [21, S. 38-41]. 
dest verbunden - auch das Konzept der „schuldhaften Verwahrlosung“.

\section{Korrespondenzadresse}

\section{MMag. phil. Ina Friedmann}

Wissenschaftsbüro Innsbruck e.V. Innsbruck, Österreich

ina.friedmann@uibk.ac.at

MMag. phil. Ina Friedmann, geb. 1985. Diplomstudien klassische Philologie und Geschichte. Doktoratsstudium Geschichte an der Universität Wien, Schwerpunkt Zeit- und Medizingeschichte. Seit 2014 wissenschaftliche Mitarbeit in (medizin)historischen Projekten an der Universität Innsbruck, darunter zur Innsbrucker Kinderbeobachtungsstation von Maria Nowak-Vogl (2014-2016) sowie zu Zwangssterilisationen in Tirol und Vorarlberg (2017-2020). Aktuell wissenschaftliche Mitarbeiterin (Wissenschaftsbüro Innsbruck) im Forschungsprojekt Studie zu Martinsbühel - Fremdunterbringung in konfessionellen Heimen in Tirol nach 1945.

\section{Einhaltung ethischer Richtlinien}

Interessenkonflikt. I. Friedmann gibt an, dass kein Interessenkonflikt besteht.

Für diesen Beitrag wurden von der Autorin keine Studien an Menschen oder Tieren durchgeführt. Für die aufgeführten Studien gelten die jeweils dort angegebenen ethischen Richtlinien.

\section{Literatur}

1. Ash MG (2002) Wissenschaft und Politik als Ressourcen füreinander. In: Bruch R, Kaderas B (Hrsg) Wissenschaften und Wissenschaftspolitik. Bestandsaufnahmen zu Formationen, Brüchen und Kontinuitäten im Deutschland des 20 Jahrhunderts. Franz Steiner, Stuttgart, S32-51

2. Asperger H (1974) Allgemeine Problematik der Heilpädagogischen Station. In: Asperger H, Lankers-Dunhofer M, Morgenbesser G, Turnowsky C (Hrsg) Zehn Jahre heilpädagogische Station der SOS-Kinderdörfer. Wagner, Innsbruck, S7-40

3. Asperger H (1952) Heilpädagogik. Einführung in die Psychopathologie des Kindes für Ärzte, Lehrer, Psychologen und Fürsorgerinnen. Springer, Wien

4. Asperger H (1942) „Jugendpsychiatrie“ und "Heilpädagogik“. Münchener Med Wochenschr 89:352-356

5. Asperger H (1946) Schwester Viktorine Zak. Erziehung Unterr 96:155-158

6. Bandhauer-Schöffmann I (2012) Der "Christliche Ständestaat" als Männerstaat? In: Tálos ENW (Hrsg) Austrofaschismus. Politik - Ökonomie - Kultur 1933-1938. LIT, Wien, S254-280

7. Dietrich-Daum E (2018) Über die Grenze in die Psychiatrie. Südtiroler Kinder und Jugendliche auf der Kinderbeobachtungsstation von Maria Nowak-Vogl in Innsbruck 1954-1987. Wagner, Innsbruck

8. Dietrich-Daum E, Ralser M, Rupnow D (2020) Psychiatrisierte Kindheiten: Die Innsbrucker Kinderbeobachtungsstation von Maria NowakVogl. Studienverlag, Innsbruck, Wien, Bozen
9. Fadinger B (1999) Die vergessenen Wurzeln der Heilpädagogik. Erwin Lazar und die Heilpädagogische Station an der Universitäts-Kinderklinik in Wien (Diplomarbeit). Universität Wien

10. Foucault M (2013) Die Anormalen. Vorlesungen am Collège de France (1974-1975). Suhrkamp, Frankfurta.M.

11. Foucault M (2014) Sexualität und Wahrheit: Erster Band: Der Wille zum Wissen. Suhrkamp, Frankfurt a.M.

12. Foucault M (2014) Überwachen und Strafen. Die Geburt des Gefängnisses. Suhrkamp, Frankfurt a.M.

13. Friedmann I (2017) Von der "Schuld“ der Opfer. Die Behandlung von Kindern, die Opfer sexueller Gewalt wurden, an der Heilpädagogischen Abteilung der Wiener Universitäts-Kinderklinik im 20. Jahrhundert. http://www.zeitgeschichte-online.de/ thema/von-der-schuld-der-opfer. Zugegriffen: 10. Apr. 2020

14. Geisthövel A, Hess V (2017) Handelndes Wissen: Die Praxis des Gutachtens. In: Geisthövel A, Hess V (Hrsg) Medizinisches Gutachten. Geschichte einer neuzeitlichen Praxis. Wallstein, Göttingen, S9-39

15. Goffman E (2016) Über die soziale Situation psychiatrischer Patienten und anderer Insassen. Suhrkamp, Frankfurta.M.

16. Lazar E (1923) Die heilpädagogische Abteilung der Kinderklinikin Wien. Z Kinderforsch 28/2:161-174

17. Lazar E (1925) Medizinische Grundlagen der Heilpädagogik. Für Erzieher, Lehrer, Richter und Fürsorgerinnen. Springer, Wien

18. Lazar E (1913) Psychische Abnormitäten bei Fürsorgezöglingen. Wien Klin Wochenschr 29:1227-1230

19. Löscher M (2005) „... der gesunden Vernunft nicht zuwider ..."? Eugenik in katholischen Milieus/ Netzwerken in Österreich vor 1938 (Dissertation). Universität Wien

20. Malleier E (2014) „Kinderschutz" und „Kinderrettung". Die Gründung von freiwilligen Vereinen zum Schutz misshandelter Kinder im 19. und frühen 20. Jahrhundert. Studienverlag, Innsbruck, Wien, Bozen

21. Ralser M, Bischoff N, Guerrini F, Jost Ch, Leitner U, Reiterer M (2017) Heimkindheiten. Geschichte de Jugendfürsorge und Heimerziehung in Tirol und Vorarlberg. Studienverlag, Innsbruck, Wien, Bozen

22. Sieder R (2018) Wissenschaftliche Diskurse, Jugendfürsorge und Heimerziehung in Wien im 20. Jahrhundert. Virus 17:29-56

23. Zak V (1928) Die Entwicklung der klinischen Heilpädagogikin Wien. Int Counc Nurses 4:348-354

24. Zak V (1932) Die Heilpädagogische Abteilung unter Lazar. Eos. Z Heilpädagogik 2:38-40 